\title{
ENSEÑANZA DE LAS TIC PARA EL DESARROLLO DE COMPETENCIAS TECNOLÓGICAS EN DOCENTES DE EDUCACIÓN BÁSICA ALTERNATIVA
Teaching of ICT for the development of technological competencies at alternative basic education teachers

\author{
Rosario Luján Alcarraz ${ }^{1}$ \\ Centro de Educación Básica Alternativa “Glorioso Húsares de Junín”, Perú
}

Recibido: 16-02-2016

Aceptado: 23-06-2016

\section{RESUMEN}

Esta investigación supuso que las innovaciones tecnológicas dependen del conocimiento y dominio que los docentes tengan de las TIC y repercuten en el trabajo pedagógico. Por ello, dar respuesta a la pregunta ¿en qué medida el Programa de Enseńanza de TIC mejora el desarrollo de las competencias tecnológicas en los docentes de los Centros de Educación Básica Alternativa (CEBA) de la Red $N^{\circ} 03$ - UGEL 05 - San Juan de Lurigancho? Tuvo como objetivo general: determinar el efecto del programa de enseñanza de TIC en el desarrollo de las competencias tecnológicas en los docentes de los referidos CEBA y consideró la hipótesis de que el Programa de Enseñanza de TIC mejora significativamente el desarrollo de las competencias tecnológicas de los docentes. Para verificar esta hipótesis se adoptó un enfoque cuantitativo, con alcance explicativo y un diseńo pre-experimental, con preprueba-posprueba y un solo grupo (experimental). Se ejecutaron una serie de talleres para desarrollar las competencias tecnológicas de los 22 docentes que conformaron la muestra. A estos se les administró una prueba estandarizada, compuesta por 20 ítems que midieron las competencias instrumentales y cognitivas. El resultado del contraste de la hipótesis general dio $\mathrm{p}=, 000$ para la diferencia entre las medias del pre test $(6,55)$ y post test $(15,45)$, evidenciándose que el Programa de Enseñanza de TIC mejora significativamente las competencias tecnológicas en los docentes de los CEBA.

Palabras Clave: Enseñanza, innovaciones pedagógicas, competencias tecnológicas, instrumentales y cognitivas, tecnologías .

\section{Abstract}

This research meant that technological innovations depend on the knowledge and mastery that teachers have about ICT, which affect the pedagogical work. Therefore, we tried to answer the question: to what extent the ICT Teaching Program improves the development of technological competencies in the Alternative Basic Education Centers (CEBA) teachers belonging to the Network No. 03 - UGEL 05 - San Juan de Lurigancho? It had as general objective: to determine the effect of the ICT Teaching Program in the development of technological competencies in the teachers

1 Magister en Administración de la Educación. Licenciada en Educación Secundaria. Especialidad en Lengua y Literatura. Docente del Área de Comunicación Integral. Directora del CEBA “Glorioso Húsares de Junín”. Con estudios de Especialización Pedagógica de los Centros de Educación Básica Alternativa, Universidad de Valencia - España. e-mail: rosariola_10@gmail.com 
of the previously mentioned CEBA and considered the hypothesis that the ICT Teaching Program significantly improves the development of technological competencies of the CEBA teachers. To verify this hypothesis a quantitative approach with explanatory scope and a pre-experimental design with pretest-posttest and only one group (experimental) was adopted. A series of workshops were implemented to develop the technological skills of 22 teachers who formed the sample. They were administered a standardized test, consisting of 20 items that measured the instrumental and cognitive competencies. The result of the contrast of the general hypothesis was $\mathrm{p}=.000$ for the difference between the average of pretest (6.55) and posttest (15.45), showing that the ICT Teaching Program significantly improves the technological competencies of CEBA teachers.

Keywords: Teaching, pedagogical innovations, technological, instrumental and cognitive competencies, technologies.

\section{INTRODUCCIÓN}

Las tecnologías de la información y comunicación (TIC), han generado una transformación en los roles de docentes y estudiantes, por lo que los sistemas educativos de todo el mundo están obligados a adaptarse a los cambios que estos han producido en las diferentes áreas del conocimiento humano, lo cual no es ajeno a aquellos profesionales que imparten cátedra tanto a nivel básico como superior, ya que el éxito de cualquier innovación pedagógica dependerá de los docentes y del nivel de conocimiento que estos tengan acerca del uso de las TIC, en esta era del conocimiento y de la gestión y apropiación de las tecnologías, para lo cual se torna necesario el desarrollo de competencias digitales, como lo demuestran investigaciones realizadas en Europa, básicamente en España, tal como refieren Esteve, Adell y Gisbert (2014) sobre:

[...] la competencia digital que deben desarrollar los docentes no solo supone la capacidad de movilizar los conocimientos, habilidades y actitudes para utilizar las TIC de manera eficiente y eficaz, sino también para mejorar y transformar las prácticas de aula, enriquecer su propio desarrollo profesional y su identidad, así como la de sus estudiantes. (p. 36)

Por su parte Sánchez, Olmos y García-Peñalvo (2016) resaltan la importancia de la integración de las nuevas tecnologías en el proceso de enseñanza aprendizaje y la aceptación de las mismas por parte de los docentes, quienes aprenden a uti- lizar nuevas estrategias y diseños didácticos en las sesiones de aprendizaje, permitiendo la apertura de realizar cambios en su quehacer pedagógico.

En Perú, todavía es incipiente el desarrollado de políticas educativas orientadas a la implementación y utilización de las TIC en los programas de formación docente, lo cual también se extrapola a los docentes en el desempeño de sus funciones, a pesar de que en el proyecto Estándares UNESCO de Competencia en TIC para Docentes (2008) hay orientaciones y directivas que amplían la formación profesional docente con miras al desarrollo de sus competencias pedagógicas, sus capacidades de cooperación y liderazgo y la utilización de las TIC en el aula. Tampoco existe una metodología de enseñanza unificada a nivel nacional, y menos con relación a la utilización de las TIC en el proceso de aprendizaje-enseńanza. Muchas veces se cree que el uso de las TIC en educación limita, sustituye o relega al profesor en su labor docente. Si a esto se le agrega el hecho de que enseñar un curso utilizando una red informática no es lo mismo que hacerlo en la forma tradicional. Al mismo tiempo, se requiere que los docentes trabajen más para lograr la interacción entre sus estudiantes y, como consecuencia de ello, muchos docentes no están dispuestos a utilizar las TIC. Entonces, ¿cómo anda la preparación de los docentes en el manejo de las TIC? Muchos no las conocen bien, por tanto, no las saben usar; necesitan entrenamiento para saber cómo incorporarlas en su labor pedagógica. 
En los docentes de los Centros de Enseñanza Básica Alternativa (CEBA) de la Red N ${ }^{\circ} 03$ de la UGEL 05 de San Juan de Lurigancho existe la misma problemática, a pesar de ser este un centro de modalidad equivalente a la educación Básica Regular pero dirigida a adolescentes, jóvenes y adultos, los docentes no hacían uso de las TIC, en su labor pedagógica (redacción de planes de clases, registros de notas, elaboración de actas, cálculo de promedios, etcétera), menos aun cuando desarrollaban sus clases lo realizaban de una manera tradicional sin apropiarse de las TIC. La realidad descrita despertó la preocupación del investigador por experimentar con un programa de enseñanza de las TIC para desarrollar las competencias tecnológicas en docentes de la mencionada Red de CEBA del distrito de San Juan de Lurigancho; con el propósito de demostrar que la preparación adecuada de los docentes en el dominio de estas herramientas les ayuda a utilizarlas funcionalmente en el quehacer pedagógico para favorecer el aprendizaje de los estudiantes. Teniendo en cuenta lo anterior, se estableció como objetivo general determinar el efecto del programa de enseñanza de TIC en el desarrollo de las competencias tecnológicas en los docentes de los CEBA de la Red Nº3 - UGEL 05 - San Juan de Lurigancho. De este se desprendieron dos objetivos específicos: (i) Determinar el efecto del Programa de Enseńanza de TIC en el desarrollo de las competencias instrumentales en los docentes y (ii) Determinar el efecto del programa de enseñanza de TIC en el desarrollo de las competencias cognitivas en los docentes.

\section{Antecedentes}

El estudio tuvo en cuenta una serie de investigaciones previas que exploraron la utilidad e importancia de las TIC en el aprendizaje-enseñanza en países de Latinoamérica donde el problema es álgido a diferencia de Europa, donde los países tienen políticas públicas concertadas y cimentadas para la apropiación de estas tecnologías en las instituciones educativas, pero a pesar de ello todavía existen algunas deficiencias.

En España, Jiménez (2015) estudió los estándares TIC en educación en los futuros docentes de la
Facultad de Educación de la Universidad Complutense de Madrid y llegó a la conclusión de que los futuros docentes están adquiriendo el perfil del nuevo profesional de la educación, pero no tenían las competencias que indican los estándares TIC en educación, para ser competitivos en la actualidad, lo que constituye un problema para el ejercicio docente. Si se considera que los docentes deben poseer conocimientos en el manejo de las TIC, por lo menos en un nivel medio, es necesario que la universidad desarrolle adecuadamente esas competencias profesionales, habiendo ya políticas publicas establecidas por el gobierno.

En Colombia, Muñoz, (2012) estudió la apropiación, uso y aplicación de las TIC en los procesos pedagógicos y comprobó que hubo una mejoría con cierta disposición al cambio de las actitudes negativas de los docentes hacia el manejo e implementación de las TIC en su labor pedagógica y en el proceso de aprendizaje de los estudiantes. El completo conocimiento de las herramientas TIC y la correcta utilización en el quehacer pedagógico de los docentes beneficia el aprendizaje de los estudiantes. No solo se trata de usar las TIC en la labor docente, sino también para lograr que los estudiantes alcancen sus logros de aprendizaje.

En Ecuador, Cueva (2012) comprobó que para los estudiantes y docentes, la utilización de las TIC en el proceso de enseñanza es muy importante; pero, especialmente los docentes, deben adquirir mayor dominio y conocimiento de las herramientas y recursos que ofrecen estas herramientas en el aprendizaje, para que las usen en forma adecuada y operativa en el trabajo pedagógico. En México, Rendón (2012) analizó la incorporación de las tecnologías en el aula y llegó a la conclusión de que la incorporación de la tecnología al aula genera oportunidades para la renovación de las relaciones en la misma, las formas de buscar y representar conocimientos y significados y prácticas docentes para el trabajo con los diferentes contenidos académicos.

Considerando estas experiencias previas y de acuerdo a criterios estandarizados, el estudio contó con una programación de unidades didácticas a través de una serie de talleres, en los cuales se integró la variable independiente referida al "Pro- 
grama de enseñanza de TIC”, la misma que a continuación se analiza.

\section{El PROgRAMA DE ENSEÑANZA DE TIC}

Las TIC actualmente son vistas como herramientas de gestión del conocimiento y facilitadoras eficaces de la comunicación global (Ministerio de Educación Nacional, 2013, p. 19). Como tal, mejoran las oportunidades de aprendizaje presentes, abriendo nuevas puertas a la construcción del conocimiento científico. Enriquecen la experiencia cultural, tecnológica y científica de quienes las usan para incrementar su bagaje cultural. En ese contexto, los programas de enseñanza de las TIC son oportunidades de invaluable trascendencia para enseñar a utilizar herramientas que facilitan el autoaprendizaje y derribar los muros que circundan a las aulas de clase tradicionales.

Un programa de enseñanza es una experiencia educativa, organizada dentro de una institución educativa, que consiste en un conjunto de cursos o módulos educativos coherentes, agrupados y ordenados en serie, los cuales proporcionan conocimientos en un área determinada, acordes al nivel propuesto, y que contienen una columna vertebral básica de los aspectos que la definen como tal. Asimismo, el programa considera el desarrollo de las habilidades básicas necesarias y la formación de actitudes para demostrar competencia y valores en la aplicación en forma adecuada del conocimiento en las diferentes expresiones de la práctica del curso programado (Proyecto Tuning - Europa, 2004, p. 25). Por su parte, para la Ley General de Educación (2012) los programas de enseñanza se organizan a través de currículos; conceptualizándolo de la siguiente manera:

Art. $33^{\circ}$. El currículo de la Educación Básica es abierto, flexible, integrador y diversificado. Se sustenta en los principios y fines de la educación peruana.

Art. $34^{\circ}$. El currículo es valorativo en tanto responde al desarrollo armonioso e integral del estudiante y a crear actitudes positivas de convivencia social, democratización de la sociedad y ejercicio responsable de la ciudadanía.
El currículo es significativo en tanto toma en cuenta las experiencias y conocimientos previos y las necesidades de los estudiantes. El proceso de formulación del currículo es participativo y se construye por la comunidad educativa y otros actores de la sociedad; por tanto, está abierto a enriquecerse permanentemente y respeta la pluralidad metodológica.

Desde esta perspectiva, el Programa de enseńanza de TIC se define como aquel que permite al profesorado desarrollar sus competencias tecnológicas para que utilice de manera eficaz y eficiente los nuevos instrumentos tecnológicos que constituyen las TIC en sus actividades profesionales (docentes, de investigación, de gestión) y personales (Marqués, 2005). Por ello, en el presente estudio se realizó la planificación curricular del programa, entendiéndose a este como "el acto de anticipar, organizar y decidir cursos variados y flexibles de acción que propicien determinados aprendizajes para el desarrollo de competencias" (MINEDU, 2015, p. 5). El Programa de enseñanza de TIC se realizó mediante una serie de talleres dirigidos a los docentes participantes, y que se describen en la tabla 1 .

Tabla 1

Descripción de talleres impartidos a los docentes participantes

\begin{tabular}{ll}
\hline Dimensiones & \multicolumn{1}{c}{ Indicadores } \\
\hline Taller & - Utiliza las herramientas de Windows \\
la computa- & e identifica los componentes de una \\
dora" & computadora. \\
& - Administra los archivos y carpetas, \\
& y se conecta a una red informática. \\
& - Configura el entorno de la compu- \\
& tadora según los requerimientos del \\
& trabajo. \\
& - Intercambia información con usua- \\
& rios de otras redes educativas. \\
& - Imprime documentos a través de \\
& impresoras conectadas en red. \\
\hline Taller & Describe los elementos del Word y \\
"Elaboración & explica la utilidad de cada uno. \\
de docu- & Da el formato a un documento \\
mentación & pedagógico. \\
pedagógica & Redacta con el formato apropiado, \\
en Word & informes, solicitudes, programas \\
& curriculares, proyectos y reglamen- \\
& tos pedagógicos. \\
\hline
\end{tabular}




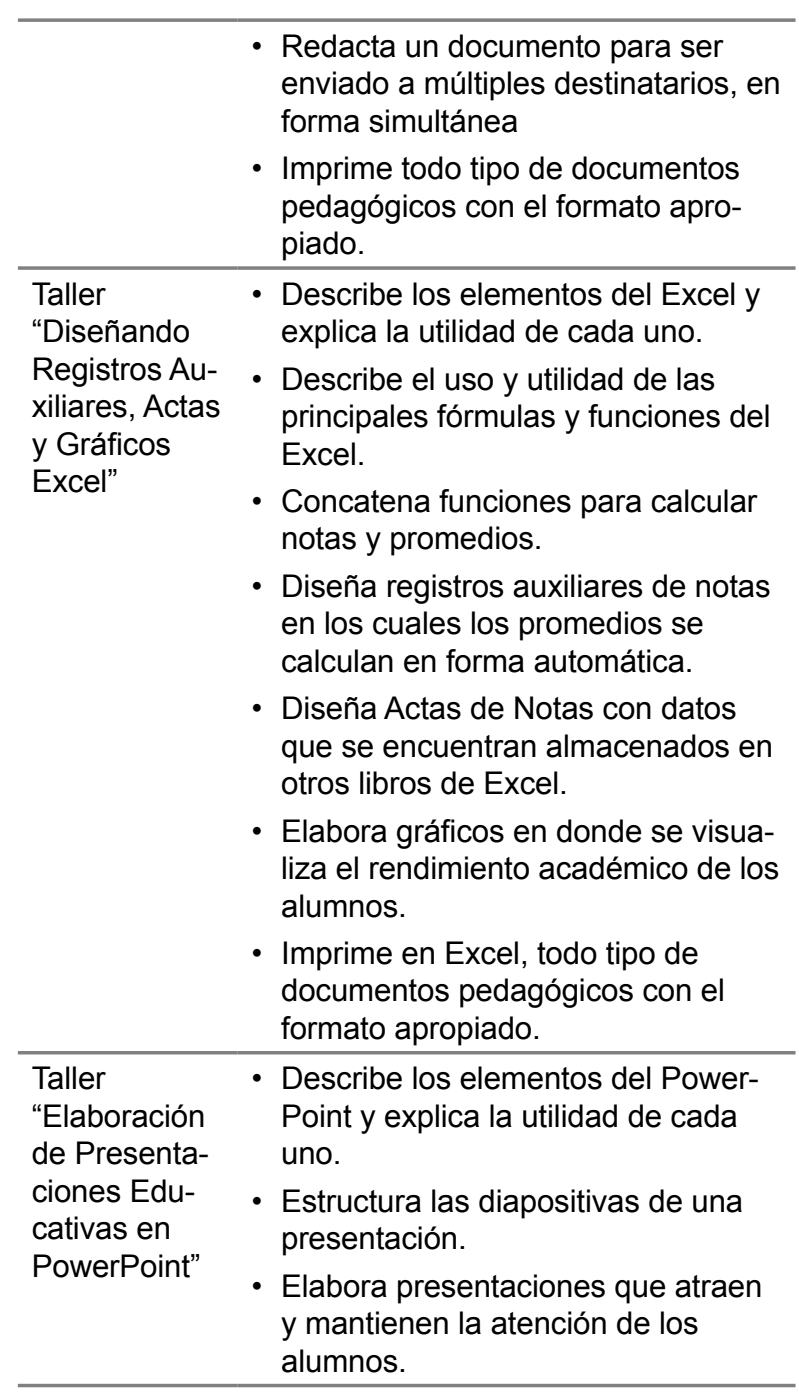

Fuente: Elaboración propia.

\section{Competencias tecnológicas}

Desde el siglo pasado, el tema sobre el desarrollo de competencias, tanto en estudiantes y docentes, ha sido investigado desde diferentes vertientes, sobre todo en el aspecto pedagógico; pero con el advenimiento de las TIC a nivel global, estas han tenido un cambio sustancial. En la actualidad se considera que no solo es analfabeto aquel que no sabe leer, sino también aquellos que no se apropian de las tecnologías en su quehacer cotidiano, más aún en su desempeño profesional; no están exentos los docentes de nivel básica, quienes forman a esta nueva generación $Z$, quienes se nutren de las tecnologías y viven de y para ella. Así refieren Fernández Cruz y Fernández Díaz (2015) cuando hablan de las altas capacidades de los estudiantes de la generación tecnológica o generación Z. Por esta razón en esta investigación se conside- ró como variable dependiente al desarrollo de las competencias tecnológicas en los docentes de los Centros de Educación Básica Alternativa.

El Ministerio de Educación Nacional (2013) señala que la innovación tiene su punto de partida en acciones de investigación específicas que tengan por finalidad construir respuestas, elaborar planteamiento renovadores y construir modelos de trabajo para cuestionar los esquemas vigentes educativos vigentes (p. 16). La innovación es pues un camino estratégico necesario en las comunidades educativas, y que pasa por la iniciativa de introducir cambios en educación e, implícitamente, desarrollar las competencias tecnológicas de docentes y estudiantes.

Por lo mencionado, se debe tener claro el término "competencia" debido a las diversas acepciones que posee, para lo cual se torna relevante vincular el contexto en que se encuentre involucrada. Según Hoyos (2013), las competencias que se van desarrollando en los estudiantes, son claves para que ellos se desarrollen plenamente, desde un ámbito social, personal y profesional, teniendo en cuenta las exigencias de la sociedad. Por ende, esto permite afirmar que ser competente implica dominar ciertos conocimientos, habilidades y una amplia variedad de saberes o recursos (Ministerio de Educación, 2014, p. 1). Por su parte, el Proyecto Tuning - Europa (2004) y Mora-Valentín y Ortíz-De Urbina (2015) hablan de tres tipos de competencias: i) básicas, que es el conjunto de conocimientos, habilidades, actitudes y valores mínimos necesarios que debe poseer todo profesional, ii) específicas, como el conjunto de conocimientos, habilidades, actitudes y valores que están vinculados a condiciones y áreas específicas de ejecución de una determinada disciplina, dentro de la cual se encuentran las competencias cognitivas, metodológicas, laborales y sociales y ii) genéricas, que son el conjunto de conocimientos, habilidades, actitudes y valores que permiten desempeñarse en una disciplina y que se adquieren independientemente de los estudios que se cursen. Para IBERTIC (s.f.), el desarrollo de una competencia no solo involucra el aspecto pedagógico, sino que incluye el uso de las tecnologías. Es 
otro tipo de habilidad que comprende un aprendizaje nuevo de conocimientos, adicionales a las áreas curriculares ya implementadas y la competencia a desarrollar debe centrarse en el uso reflexivo y creativo de las herramientas tecnológicas. La OCDE (s.f.), explica que la modernización y globalización han generado un mundo más diverso e interconectado, con dominio de tecnologías cambiantes, por lo que las competencias a desarrollar deben ser más definidas y apuntar a estas nuevas tendencias, para la experticia en el manejo, uso y apropiación de estas herramientas tecnológicas. El desarrollo de competencias tecnológicas, entendidas como la utilización de herramientas o recursos de manera interactiva, la actuación autónoma y el accionar en grupos socialmente heterogéneos. Por su parte, Almerich, Suárez et al. (2010, p. 33) refiere que los docentes deben considerar dimensiones fundamentales de la competencia tecnológica, como: a) tecnología básica, incluida en ella la terminología y uso del sistema operativo, b) las aplicaciones informáticas básicas, como el procesador de texto, hoja de cálculo y base de datos, c) aplicaciones multimedia, d) presentaciones multimedia, e) software educativo, f) conocimientos sobre Internet, navegación, comunicación como uso del correo electrónico, foros, chat, búsqueda de información y elaboración de páginas web.

Gewerc et al. (2011, p. 23) refiere que existe "la competencia informática relacionada a la capacidad que tienen los individuos para utilizar de manera adecuada las TIC vinculadas al ordenador, los programas informáticos y la red, y la competencia informacional, es que permite capacitar para buscar, seleccionar, evaluar y comunicar la información”. Bielva et al. (2015) resalta que las competencias informacionales, en las que se encuentran inmersas: (i) la búsqueda de información, implica el rastreo planificado de información específica, de acuerdo con un objetivo determinado; (ii) el procesamiento de información, que consiste en la lectura y comprensión del material informativo, con la respectiva superación de las dificultades que se presenten durante el proceso, precisando que en esta los docentes deben trabajar más, pues el procesamiento adecuado de la información permitirá seleccionar el contenido más idóneo para su trabajo pedagógico y (iii) la comunicación y difusión de información, cuyo desarrollo permitirá al docente saber comunicar con la mayor claridad y sencillez posible la información seleccionada, logrando que los receptores de la misma comprendan exactamente lo que se les quiere hacer saber. Hay una interdependencia e interrelación entre estas competencias, que son muy importantes para el futuro del profesorado (Martínez-Abad et al., 2015); su dominio se traduce en la calidad del desempeño de los docentes. Desde el punto de vista de Amador et al. (2015, p. 89), las competencias tecnológicas permiten una apropiación de las TIC, el manejo y destreza para navegar, apropiarse de las Web 2.0 y 3.0 y defenderse en un mundo tecnológico; su aprovechamiento hace más cómoda la vida y contribuye a la calidad educativa.

Desde esta perspectiva, el docente como guía, facilitador o tutor no solo debe de poseer competencias pedagógicas inherentes a su labor, sino debe de estar a la par del avance de las TIC, por lo que se hace imprescindible que desarrolle competencias tecnológicas, digitales o informacionales en su quehacer cotidiano dentro y fuera el aula.

\section{Materiales y Métodos}

Las variables estudiadas son el programa de enseñanza de las TIC (X) y competencias tecnológicas en docentes $(\mathrm{Y})$. El trabajo realizado analizó el efecto del programa en el desarrollo de las competencias tecnológicas de los docentes de los Centros de Educación Básica Alternativa (CEBA). En las secciones siguientes se detalla el método de trabajo utilizado para verificar el efecto de la variable $\mathrm{X}$ en la variable $\mathrm{Y}$.

\section{Tipo y Diseño}

La investigación es de tipo aplicada o tecnológica, pues tuvo por finalidad mejorar las competencias tecnológicas de los docentes mediante la ejecución del programa de enseñanza de TIC (Mejía, 2005a, p. 33). Se desarrolló con un enfoque cuantitativo y alcance explicativo. Los datos se recogieron con base en una escala de medición numérica, para analizarlos mediante la estadísica descriptiva e inferencial. Se utilizó un diseño pre-experimental, con preprueba-posprueba y un 
solo grupo (experimental). Se trabajó con grupos intactos, es decir, los sujetos no se asignaron al azar, el grupo ya estaba formado antes del experimento (Hernández et al., 2010).

\section{Participantes}

La poblacion estuvo formada por los CEBA que cuentan con apropiados laboratorios de cómputo para que los docentes realicen las prácticas de los talleres programados para el desarrollo de competencias tecnológicas. Por tal razón, fueron seleccionados los CEBA "Albert Einstein y Julio C. Tello", integrantes de la Red $N^{\circ} 03$ de la UGEL 05. La muestra fue de tipo no probabilística, intencional y estuvo conformada por la totalidad de profesores (22), quienes provenian de estratos socioeconómicos diversos. El rango de edad fue de 25 a 40 años, de ambos géneros.

\section{Instrumentos}

La recopilación de los datos se realizó a través de la técnica de prueba estandarizada, que según Hernández et al. (2010) y Fernández (2013) , evalúa actitudes, proyecciones y proporciona información sobre variables medidas mediante expresiones escritas. El instrumento utilizado para medir el nivel de desarrollo de las competencias instrumentales y cognitivas en el uso de las TIC fue la prueba de "Verificación de la aplicación de las TIC, en la labor docente de los CEBA". Constaba de 20 ítems (competencias instrumentales = $10 \mathrm{y}$ competencias cognitivas $=10$ ). La calificación tuvo en cuenta los niveles de logro señalados por el Ministerio de Educación (MINEDU, 2005, 2009), y que se muestran en la siguiente tabla.

\section{Tabla 2}

Niveles de logro

\begin{tabular}{cc}
\hline Calificación & Rango \\
\hline AD & $20-18$ \\
A & $17-14$ \\
B & $13-11$ \\
C & $10-00$ \\
\hline
\end{tabular}

Fuente: Ministerio de Educación (2005, 2009).
Para verificar la confiabilidad de la prueba, se realizó una administración piloto con 10 docentes que ya aplicaban el uso de TIC en su quehacer pedagógico. Posteriormente se calculó el índice de confiabilidad mediante el coeficiente de confiabilidad de Küder-Richardson (Mejía, 2005b, p. 29), que fue $\mathrm{Cf}=0,67$. De esta manera se demostró que la prueba era muy confiable y podía utilizarse en la investigación. Asimismo, se verificó el grado de dificultad, calculado en 59\% (dificultad adecuada). Estas evidencias se complementaron con la opinión de 5 expertos en el tema, quienes otorgaron una calificación promedio de $91 \%$, dejando constancia de que el instrumento podía emplearse en la investigación. La administración de la prueba fue de manera individual, en un tiempo promedio de 80 minutos, usando las PC de los laboratorios de cómputo de los CEBA.

Tabla 1

Descripción de talleres impartidos a los docentes participantes

\begin{tabular}{|c|c|c|}
\hline Dimensiones & Indicadores & Ítems \\
\hline \multirow{3}{*}{$\begin{array}{l}\text { Prueba de } \\
\text { Verificación } \\
\text { de la aplica- } \\
\text { ción de las } \\
\text { TIC, en la } \\
\text { labor docente } \\
\text { de los CEBA }\end{array}$} & $\begin{array}{l}\text { a. Conocimientos y } \\
\text { utilización de los equipos } \\
\text { informáticos. }\end{array}$ & $1-5$ \\
\hline & $\begin{array}{l}\text { b. Conocimiento y uso } \\
\text { funcional y creativo de los } \\
\text { programas informáticos } \\
\text { estándar }\end{array}$ & $6-10$ \\
\hline & $\begin{array}{l}\text { c. Aplicación de las tecnolo- } \\
\text { gías de la información en } \\
\text { el trabajo administrativo } \\
\text { docente. }\end{array}$ & $11-20$ \\
\hline
\end{tabular}

Fuente: Elaboración propia.

\section{Confidencialidad}

Aunque la participacion fue voluntaria, se solicitó el consentimiento de los participantes, garantizándoles la protección de su identidad, en respeto de sus derechos individuales. De esta manera, la información obtenida cuenta con las garantías del caso y los resultados son producto del análisis objetivo y riguroso de los datos. (APA, 2010).

\section{Procedimiento}

La realización del estudio involucró 4 etapas, las mismas que se detallan a continuación: 
Primera etapa: Búsqueda de información bibliográfica y antecedentes. Para tener un fundamento teórico de la problemática detectada se realizó una búsqueda general en la base de datos de Concytec, Repositorios de acceso libre a nivel internacional a revistas y bibliotecas virtuales de universidades de reconocido prestigio.

Segunda etapa: Elaboración de materiales. Después de haber recopilado toda la información proveniente de la literatura, se planificaron las actividades a desarrollar mediante los talleres previstos y la elaboración de la prueba de verificación para el pre y post test.

Tercera etapa: Investigación de Campo. Teniendo todos los materiales elaborados y contando con un cronograma, se inició el desarrollo del programa de enseñanza TIC, previa a la autorización de los directores de los CEBA seleccionados. El trabajo tuvo una duración de cinco meses.

Cuarta etapa: Sistematización de análisis e interpretación de los resultados. Una vez concluidos los talleres, y habiéndose aplicado el post test, se analizaron e interpretaron los resultados del pre test y el post test. En el análisis estadístico descriptivo e inferencial se utilizó el programa Excel v.2010 y el software estadístico SPSS v.19 Los hallazgos se sistematizaron en tablas y gráficos estadísticos.

\section{Resultados}

El análisis inferencial para someter a contraste las hipótesis de investigación se realizó utilizando la prueba de rangos de Wilcoxon, puesto que no todos los datos de la muestra provenían de una distribución normal. Los resultados de dichas pruebas se describen a continuación.

\section{Hipótesis General}

$\mathrm{H}_{\mathrm{a}}$ : El programa de enseñanza de TIC mejora significativamente el desarrollo de las competencias tecnológicas de los docentes.

$\mathrm{H}_{0}$ : El programa de enseñanza de TIC no mejora significativamente el desarrollo de las competencias tecnológicas de los docentes de los CEBA.
Según la tabla 4, el desarrollo de las competencias tecnológicas en los docentes evidencia una mejoría luego de la ejecución del programa de enseñanza de las TIC. La media del pre test $(6,55)$ es bastante baja con relación a la media del post test $(15,45)$. La prueba de Wilcoxon dio $\mathrm{p}=0,000$, indicando que las competencias tecnológicas de los docentes participantes del programa de enseñanza de las TIC mejoraron considerablemente. La diferencia entre el pre test y el post test fue 8,9 puntos.

\section{Tabla 4}

Descripción de talleres impartidos a los docentes participantes.

\begin{tabular}{|c|c|c|c|c|c|c|}
\hline Instrumentos & $m_{0}$ & $m_{e}$ & $\mathbf{S}^{2}$ & $\bar{x}$ & Dif. & Wilcoxon \\
\hline Pre test & 6 & 6 & 1.143 & 6.55 & \multirow{2}{*}{8.9} & $W=-4.185$ \\
\hline Post test & 15 & 15 & 1.405 & 15.45 & & $p=0.000$ \\
\hline
\end{tabular}

Fuente: Prueba de verificación de la aplicación de las TIC en la labor docente de los EBA

La figura 1 grafica las diferencias entre el pre test y el post test de la prueba sobre desarrollo de las competencias tecnológicas de los docentes. La mediana del post test (14) es 8 puntos más que la mediana del pre test (6).

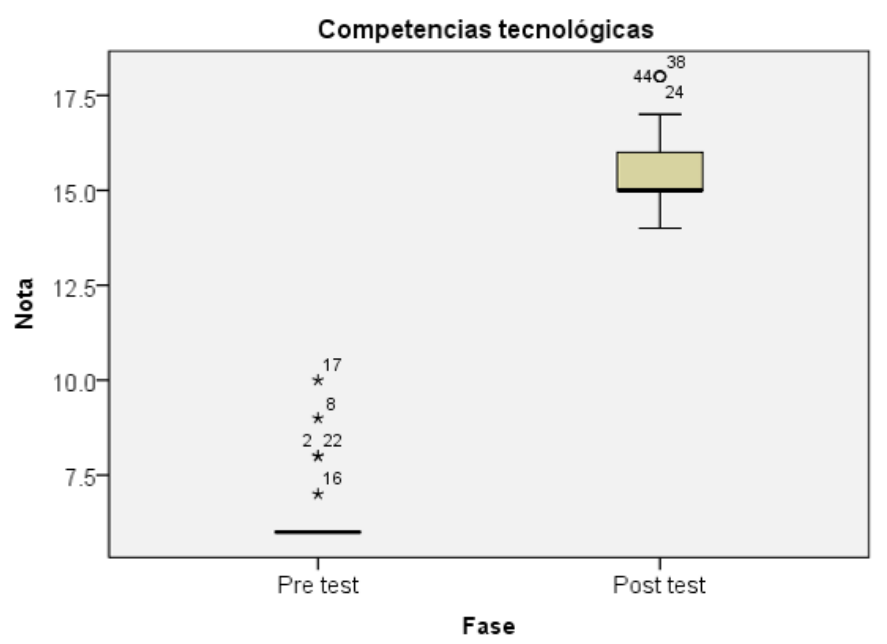

Figura 1. Comparación de las puntuaciones en el desarrollo de las competencias tecnológicas de los docentes de los CEBA, pre test y post test.

\section{Hipótesis Específica 1}

$\mathrm{H}_{\mathrm{a}}$ : El programa de enseñanza de TIC mejora significativamente el desarrollo de las competencias instrumentales de los docentes de los CEBA. 
$\mathrm{H}_{0}$ : El programa de enseñanza de TIC no mejora significativamente el desarrollo de las competencias instrumentales de los docentes de los CEBA.

La tabla 5 muestra que el desarrollo de las competencias instrumentales en los docentes evidencia una notable mejoría después de la ejecución del programa de enseñanza de las TIC. La media del pre test $(6,82)$ es 9 puntos menor que la media del post test $(15,82)$. La prueba de Wilcoxon dio $\mathrm{p}=0,000$, indicando que las competencias instrumentales de los docentes participantes del programa de enseñanza de las TIC mejoraron significativamente.

\section{Tabla 5}

Medidas estadísticas de la dimensión competencias instrumentales de los docentes de los CEBA, pre test y post test.

\begin{tabular}{|c|c|c|c|c|c|c|}
\hline Instrumentos & $m_{0}$ & $m_{e}$ & $\mathbf{S}^{2}$ & $\bar{x}$ & Dif. & Wilcoxon \\
\hline Pre test & 6 & 6 & 1.708 & 6.82 & \multirow{2}{*}{9.0} & $W=-4.226$ \\
\hline Post test & 16 & 16 & 1.368 & 15.82 & & $p=0.000$ \\
\hline
\end{tabular}

Fuente: Prueba de verificación de la aplicación de las TIC en la labor docente de los CEBA

En la figura 2 se observa la diferencia significativa entre los resultados del pre test y post test de la dimensión competencias instrumentales. La mediana del post test (16) supera en 10 puntos a la del pre test (6).

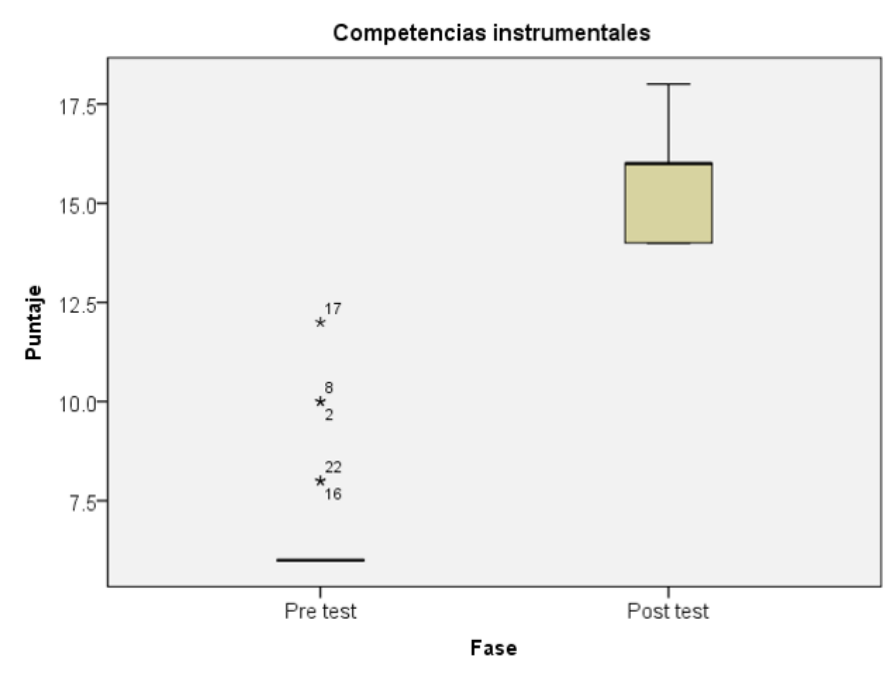

Figura 2. Comparación de las puntuaciones en la dimensión competencias instrumentales, pre test y post test. CEBA.

\section{Hipótesis Específica 2}

$\mathrm{H}_{\mathrm{a}}$ : El programa de enseñanza de TIC mejora significativamente el desarrollo de las competencias cognitivas de los docentes de los CEBA.

$\mathrm{H}_{0}$ : El programa de enseñanza de TIC no mejora significativamente el desarrollo de las competencias cognitivas de los docentes de los CEBA.

La tabla 6 muestra que el desarrollo de las competencias cognitivas en los docentes tuvo una importante mejoría después de la ejecución del programa de enseñanza de las TIC. La media del pre test $(6,27)$ es 8,82 puntos menor que la media del post test $(15,09)$. La prueba de Wilcoxon dio $\mathrm{p}=$ 0,000 , indicando que las competencias cognitivas de los docentes participantes del programa de enseñanza de las TIC también mejoraron significativamente.

\section{Tabla 6}

Medidas estadísticas de la dimensión competencias cognitivas de los docentes de los CEBA, pre test y post test.

\begin{tabular}{|c|c|c|c|c|c|c|}
\hline Instrumentos & $\mathrm{m}_{\mathrm{o}}$ & $m_{e}$ & $\mathbf{S}^{2}$ & $\bar{x}$ & Dif. & Wilcoxon \\
\hline Pre test & 6 & 6 & 0.703 & 6.27 & \multirow{2}{*}{8.82} & $W=-4.274$ \\
\hline Post test & 14 & 14 & 1.716 & 15.09 & & $p=0.000$ \\
\hline
\end{tabular}

Fuente: Prueba de verificación de la aplicación de las TIC en la labor docente de los

La Figura 3 representa la diferencia existente entre el pre test y el post test de la Dimensión Competencias Cognitivas. La mediana del post test (14) se halla por encima de la mediana del pre test (6).

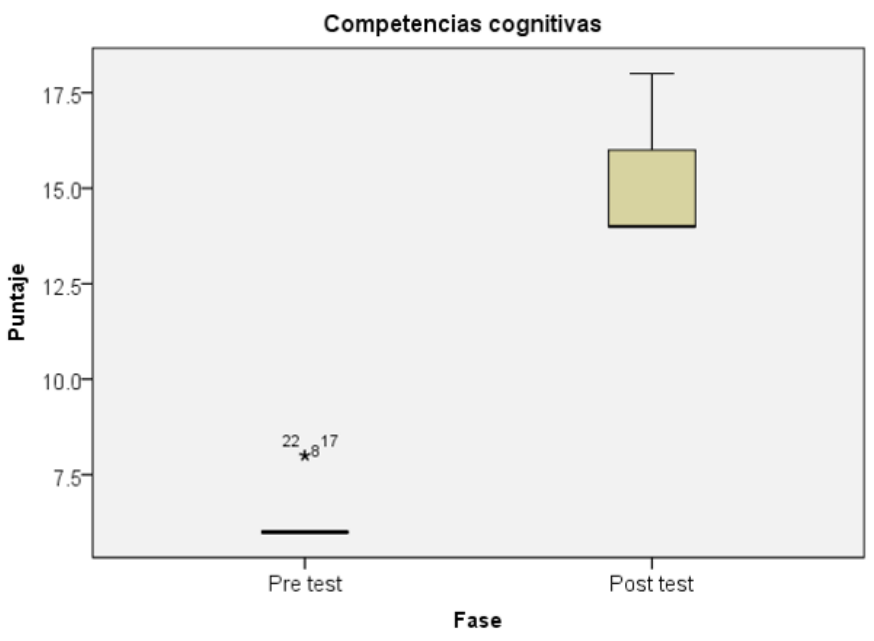

Figura 3. Comparación de las puntuaciones en la dimensión competencias cognitivas, pre test y post test. 


\section{DisCUSIÓN Y CONCLUSIONES}

El contraste de la hipótesis general permitió comprobar que la aplicación del programa de enseñanza TIC mejoró significativamente $(p=, 000)$ el desarrollo de las competencias tecnológicas de los docentes de los CEBA. Entre las mediciones del pre test y post test hubo una diferencia considerable $(8,9)$, pues el $81,8 \%$ de docentes registraron una importante mejoría en el desarrollo de sus competencias tecnológicas. Este resultado guarda relación con los hallazgos encontrados en Ecuador por Cueva (2012), quien comprobó que la utilización de las TIC en el proceso de enseñanza es muy importante tanto para docentes, como para estudiantes, ya que favorece el proceso de aprendizaje de estos últimos. Al respecto, Vargas-D'Uniam et al. (2014) señalan que los docentes reconocen ser competentes en el uso de aplicaciones informáticas como procesador de texto, hoja de cálculo y bases de datos, herramientas que utilizan en la realización de presentaciones que les ayudan a dar explicaciones más efectivas de los temas y también les permiten desarrollar su capacidad para diseñar o modificar páginas web o blogs.

El contraste de hipótesis indicó que el programa de enseñanza de TIC mejora significativamente $(p=, 000)$ el desarrollo de las competencias cognitivas de los docentes de los CEBA. El 81,8\% de docentes (mayoría) obtuvieron calificaciones de entre 14 y 17 puntos, mientras que el 18,2\% obtuvieron puntajes entre 18 y 20 puntos, elevándose considerablemente el desarrollo de las competencias instrumentales. Estas competencias tienen el carácter de herramientas procedimentales, es decir, el docente que las posee está en condiciones de utilizar las TIC en el contexto profesional y educativo para comunicarse y acceder a fuentes de información. Estos resultados coinciden con la investigación acerca de la apropiación, uso y aplicación de las TIC en los procesos pedagógicos realizada por Muñoz (2012), quien comprobó una mejoría en los docentes, comprendidos en la muestra y quienes tuvieron un cambio de actitud hacia el manejo e implementación de las TIC en su labor pedagógica y en el proceso de aprendizaje de los estudiantes.

El resultado de la prueba de hipótesis indicó que el programa de enseñanza de TIC mejora significativamente $(p=, 000)$ el desarrollo de las competencias cognitivas de los docentes de los CEBA. Hubo la evidencia de que el $77,3 \%$ de docentes (la mayoría) alcanzaron calificaciones de entre 14 y 17 puntos y solo el $22,7 \%$ obtuvo puntuaciones entre 18 y 20 puntos. Las competencias cognitivas implican un cambio de concepción acerca del aprendizaje, qué es y cómo se produce, de qué manera las nuevas tecnologías de la información y comunicación favorecen u obstaculizan el proceso de cambio. Rendón (2012), en México, concluyó que la incorporación de la tecnología en el aula genera oportunidades para la renovación de las relaciones dentro de esta, las formas de buscar y representar conocimientos y significados, como también la optimización de las prácticas docentes con respecto a los contenidos académicos. Pérez et al. (2015) refieren que el docente no solo necesita manejar las TIC, sino saber utilizarlas con fundamentos didácticos para un desempeño pedagógico de calidad, dominando los diversos recursos informáticos como apoyo a exposiciones sincrónicas o asincrónicas, búsquedas avanzadas de información y discusiones electrónicas, para beneficio propio y de sus estudiantes. Como indica Jiménez (2015), es necesario que las universidades que forman docentes los preparen competitivamente y desarrollen las competencias tecnológicas de estos; solo así estarán en condiciones de satisfacer las expectativas de los estudiantes a los que educan y forjarán una educación de calidad.

Parece ser que hay consenso en los docentes respecto a la utilidad innegable de las TIC en el proceso de aprendizaje; alcanzar un buen dominio de estas aumenta sus competencias pedagógicas. Eso mismo afirman Almerich et al. (2010), al demostrar que un $40 \%$ de los docentes poseen un nivel básico de competencia tecnológica; quiere decir que los recursos tecnológicos son un medio importante en el proceso de integración de las TIC. De igual manera, Mortis (2013, p. 147), en su investigación sobre competencias digitales en docentes de educación secundaria en el Noroeste de México, concluyó que los docentes se perciben competentes en las áreas instrumentales y cognitivas con relación al uso de las TIC, estimando como bueno el uso que le dan a las herramientas tecnológicas. 
Para futuras investigaciones, se recomienda ampliar el horizonte de las competencias tecnológicas, debido al cambio que estás han tenido. Debe considerarse no solo herramientas informáticas básicas como hojas de cálculo, procesadores de textos, Power Point, sino también herramientas más avanzadas como aplicaciones multimedia, software educativo libre, redes sociales para uso educativo, búsqueda de información, uso de bibliotecas virtuales y otras nuevos medios informáticos que la revolución tecnológica ponga al alcance de la humanidad. Igualmente, sería relevante realizar estudios comparativos entre docentes en formación y aquellos que están desempeñando su función docente relacionada al desarrollo de las competencias tecnológicas, e incluir las herramientas TIC que tienen a su disposición en sus centros de trabajo o de formación.

\section{REFERENCIAS BIBIOGRÁFICAS}

Almerich, G, Suárez, J., Belloch, C. y Orellana, N. (2010). Perfiles del profesorado a partir del conocimiento de los recursos tecnológicos y su relación con el uso que hacen de estas tecnologías. Revista Complutense de Educación, 21 (2), 247-269.

Almerich, G, Suárez, J., Orellana, N. y Díaz, M. (2010). La relación entre la integración de las tecnologías de la información y comunicación y su conocimiento. Revista de investigación educativa, 28 (1), 31-50.

Amador, J., Rojas, j., Sánchez, H. y Amador, E. (2015). Transformaciones comunicativas en el ambiente de aprendizaje de una institución beneficiaria de CPE cuando los docentes desarrolla competencias técnicas y tecnológicas e incorporan TIC en las actividad conjunta. Sientia et Technica, 20 (1), 88-94.

Asociación Americana de Psicología. (2010). Manual de Publicaciones. México, D.F.: El Manual Moderno.

Bielva, M., Martínez, F., Herrera, M. y Rodríguez, M. (2015). Diseño de un instrumento de evaluación de competencias informacionales en educación secundaria obligatoria a través de la selección de indicadores clave. En: Teoría de la Educación. Educación y Cultura en la Sociedad de la Información, vol. 16, núm. 3, septiembre, 2015, pp. 124-143.

Cueva, V. (2012). Las TICs y el desempeño docente en el colegio fiscal María Eugenia de Ruperti, del cantón Paján, provincia de Manabí, año 2012. Diseño de un sistema informático de capacitación para docentes. (Tesis de maestría inédita, Universidad de Guayaquil, Ecuador). Recuperada de: http://repositorio.ug.edu.ec/bitstream/redug/1124/1/ Las\%20Tics\%20y\%20el\%20desempe\%C3\%B1o\%20docente
Esteve, F., Adell, J. y Gisbert, M. (2014). Diseño de un entorno 3D para el desarrollo de la competencia digital docente en estudiantes universitarios: usabilidad, adecuación y percepción de utilidad. Revista Latinoamericana de Tecnología Educativa, 13 (2), 35-47.

Fernández, M. (2013). Las pruebas estandarizadas y el diseño de la política educativa en México. Recuperado de: http://www.seg.guanajuato.gob.mx/Ceducativa/CDocumental/Doctos/2013/Septiembre/Las\%20pruebas\%20estandarizadas.pdf?

Fernández Cruz, F. y Fernández Díaz, M. (2015). Los docentes de la Generación $\mathrm{Z}$ y sus competencias digitales. Comunicar, 46 (5), 97-105.

Gewerc, A., Montero, L., Pernas, E. y Alonso, A. (2011). Competencia digital y planes de estudio universitarios. En busca del eslabón perdido. Revista de Universidad y sociedad del Conocimiento (RUSC), 8 (2), 14-30. DOI: http:// dx.doi.org/10.7238/rusc.v8i2.1070

Hernández, R., Fernández, C. y Baptista, P. (2010). Metodología de la investigación. México: Mc Graw Hill.

Hoyos, L. (2013). Competencia digital docente. Albacete: Marpadal Interactive Media.

IBERTIC. (s/f). Manual para la evaluación de proyectos de inclusión de TIC en educación. Un proyecto regional de cooperación para la integración de la cultura digital en los sistemas educativos. Recuperado de: http://www.google. com.pe/url?url=http://www.ibertic.org/evaluacion/pdfs/ ibertic_manual.pdf

Jiménez, J. (2015). Estudio los estándares TIC en educación en los futuros docentes de la Facultad de Educación de la Universidad Complutense de Madrid. (Tesis doctoral inédita, Universidad Complutense de Madrid, España). Recuperada de: http://eprints.sim.ucm.es/30925/1/T36158.pdf

Ley General de Educación Nro. 28044. (2012) Recuperada de: http://www2.congreso.gob.pe/sicr/cendocbib/con4_ uibd.nsf/7978CEFD229EFE1505257A9A007083EA/\$FILE/ley_sineace_2.pdf

Marqués, P. (2005) 5 claves para una buena integración de las TIC en los centros -OEI. Recuperado de; www.oei.es/ tic/santillana/marques.pdf

Martínez-Abad, F., Olmos-Migueláñez, S., \& Rodríguez-Conde, M. J. (2015). Evaluación de un programa de formación en competencias informacionales para el futuro profesorado de E.S.O. Revista de Educación, 370, 45-70. Doi: 10.4438/1988-592X-RE-2015-370-296

Mejía, E. (2005a). Metodología de la investigación científica. Lima: Ediciones de la Universidad Nacional Mayor de San Marcos.

Mejía, E. (2005b). Técnicas e instrumentos de investigación. Lima: Ediciones de la Universidad Nacional Mayor de San Marcos.

Ministerio de Educación - MINEDU. (2005). Evaluación de los aprendizajes de los estudiantes en la educación básica regular. Recuperado de: 
http://www.minedu.gob.pe/normatividad/directivas/DIR004-2005-VMGP.pdf

Ministerio de Educación. (2009). Diseño curricular nacional de educación básica regular. Recuperado de: http://www. minedu.gob.pe/DeInteres/xtras/dcn_2009.pdf

Ministerio de Educación. (2015). Orientaciones para el uso de las unidades didácticas y sesiones de aprendizaje. Recuperado de: http://www.minedu.gob.pe/rutas-del-aprendizaje/sesiones2016/pdf/primaria/cartillas/tercer-grado/cartilla-3ro-grado.pdf

Ministerio de Educación. (2014). Orientaciones generales para la planificación curricular. La labor docente de diseñar y gestionar procesos de aprendizaje de calidad. Recuperado de: http://www.ugel06.gob.pe/agp/materiales/orientaciones-progra-ebr.pdf

Ministerio de Educación Nacional. (2013). Competencias TIC para el Desarrollo Profesional Docente. Colombia: CSNI.

Mora-Valentín, E. M., y Ortiz de Urbina Criado, M. (2015). ¿Cómo fomentar el desarrollo de competencias en la formación on-line? Una experiencia en la asignatura de dirección estratégica. Education in the Knowledge Society, 16 (2), 90108. Doi: http://dx.doi.org/10.14201/eks201516290108

Mortis, S., Valdés, A., Angulo, J., García, R. y Cuevas, O. (2013). Competencias digitales en docentes de educación secundaria. Municipio de un Estado del Noroeste de México. Perspectiva educacional, 52 (2), 135-153.

Muñoz, J. (2012). Apropiación, uso y aplicación de las TIC en los procesos pedagógicos que dirigen los docentes de la Institución Educativa Núcleo Escolar Rural Corinto. (Tesis de maestría inédita, Universidad Nacional de Colombia). Recuperada de: http://www.bdigital.unal.edu.co/6745/1/ jasminlorenamunozcampo.2012.pdf

Organización para la Cooperación y el Desarrollo Económico - OCDE. (s.f.). La definición y selección de competencias clave. Resumen ejecutivo. Recuperado de: http:// comclave.educarex.es/pluginfile.php/130/mod_resource/ content/3/DESECO.pdf

Proyecto Tuning Educational Structures in Europe. (2004) Proyecto Tuning (2004 - 2007). Recuperado de: http://tuning.unideusto.org/tuningal

Pérez, M., Ciudad, F., Farray, O., Burget, I., Piñeiro, Y. y López, J. (2015). Programa de entrenamiento en TIC como medio del proceso de enseñanza aprendizaje. Revista Cubana de ciencias informáticas, 9 (3), 138-152.

Rendón, V. (2012). La computadora llega al aula: la incorporación de las tecnologías digitales a la práctica docente. Un estudio de caso. (Tesis de maestría inédita, Centro de Investigación y de Estudios Avanzados del Instituto Politécnico Nacional, México). Recuperada de: http://www.lets. cinvestav.mx/Portals/0/SiteDocs/TesisSS/Maestria/lets_sur_ tesis_Victor_Rendon.pdf

Sánchez, J., Olmos, S. y García-Peñalvo, F. (2016). Informal tolos in formal contexts: Development of a model to assess the acceptance of mobile technologies among teachers.
Computers in Human Behavior, 519-529. Recuperado de: http://repositorio.grial.eu/bitstream/grial/521/1/Informal\%20Tools_V2.pdf

UNESCO. (2008). Estándares de competencia en TIC para docentes. Londres, disponible en: http:// www.eduteka.org/ EstandaresDocentesUnesco.php

Vargas-D’Uniam, J., Chumpitaz-Campos, Suárez-Díaz, G. y Badia, A. (2014). Relación entre las competencias digitales de docentes de educación básica y el uso educativo de las tecnologías en las aulas. Profesorado, Revista de curriculum y formación del profesorado, 18 (3), 371-377. 